\title{
IoT Based Healthcare System Model for quarantine centers Post Covid 19 Outbreak
}

\author{
Ishan Bhatt ${ }^{1}$, Shweta Yadav ${ }^{2}$ \\ ${ }^{1}$ Amity University, Noida, Uttar Pradesh, India(201311) \\ ishanbhatt.korba@gmail.com \\ ${ }^{2}$ Amity University, Noida, Uttar Pradesh, India(201311) \\ shweta.ece@gmail.com
}

\begin{abstract}
The bright capability of showing up Internet of things (IoT) advancements for wellbeing gadgets and sensors assumed an essential job in the new age of Healthcare industry for secure patient care and maintaining social distancing. After Corona virus outbreak, it has been observed that there is an utter need for the automated healthcare system for the patients in need or elderly incapable or un-helped people. This paper proposed a Healthcare and quarantined install with IoT devices for the benefiting of quarantined people and Covid-19 patients.
\end{abstract}

Keywords-HealthIIoT, body sensor network, Low power network, HYDRA middleware, health care model post covid19, IoT based quarantine centers

\section{Chapter 1. Introduction}

After corona virus outbreak, hundreds of thousands of people are quarantined and their health is being constantly monitored. As it is essential to maintain social distancing but due to regularly monitoring the health parameters of patients by medical staff. Members of medical staff are at huge risk to getting corona virus. By saluting to their determination, this paper attempts to create a new model for gathering vital information of patients without putting members of medical teams putting at great risk. This model is made cost effective to ensure wide adaptability and less maintenance cost. It is also taken care that these equipment's is made with copper.

IoT platform is an ambitious approach in Healthcare Industry, which effectively connects body sensor network with the communication network. In order to manage the complexity of such systems, interworking solutions that can reuse established technologies seamlessly with healthcare network. From choosing of right sensors to sending the error free patient data with the help of right wireless connectivity technology can be relatively challenging to healthcare industry [1]. IoT to healthcare industry provide automation and analytics system, which utilizes sensing, networking, data and technology to deliver complete HealthIIoT system. The high flexibility and ability to work in any infrastructure enhances the application of IoT in various other industries as well.
One of the challenges of Health Industry are the distribution of accurate and real time information of patients. IoT application in healthcare industry enhances facilities and professional practice it brings it out into the home, office, or social space. It allows individual in monitoring to their own health and allow service provider to deliver better treatment. However, there are many other challenges in Designing an IoT based healthcare system includes security, authentication and exchanging data.

Many research scholars simply overlook the cast of machines and keep on inventing and discovering costly technology which makes it less possible to become advantageous to society. The models and channel proposed in these papers were strategically created keeping cost in mind.

After corona virus outbreak lives of many doctors and medical staff are at high risk. It is high time for governments to adopt technologies that help medical staff to maintain safe distance from patients.

\section{CHAPTER 2: RELATED WORK DONE}

In the paper [1] LED based PPG sensors are used to measure heart beats. Nasal sensor which is used for respiratory rate count are used in paper [5][6] based on thermistor is also highly sensitive to another source of temperature, although this technique works well. ECG enable respiratory sensor used in [7] to obtain respiratory rate, but it is not a long wearable as causes skin allergies. In paper [6] author used microphone but this is also highly inflicted by external noises. Fibreoptic sensor used elastic substrate in [7] here author suggested that it is $95 \%$ accurate and long-time wearable but has also susceptible to noise

In body area network there is a central body(node) which act as a centre of the star topology with all sensors linked to it and seamlessly communicate data. In paper [13] author suggests BLE communicate up to $150 \mathrm{~m}$ in an open area, sufficient for healthcare system WBAN. The major advantage is that it has a low latency(Delay) rate of only $3 \mathrm{~ms}$ and it is discussed in paper [15]. It provides high data rate of 1 Mbps. 
Website: ijetms.in Issue:4, Volume No.4, July-2020 DOI: 10.46647/ijetms.2020.v04i04.011

In [15] for long distance communication author suggested that HealthIIoT over other two standards of LPN.NB-IoT can operate in LTE bands. Due to high receiver sensitivity it covers the area up to $15 \mathrm{~km}$ with high speed explained in paper [16]. 3GPP(S3) protection scheme is used by NB-IoT including other security features like authentication, securing user and device identity and data integrity proposed in paper [17]. All the technologies and limitations are mentioned in [21]. A detailed explanation of long-distance health monitoring in [22].

\section{Chapter 3. Body Sensor Network}

These wearable sensors will be given to patients when they are quarantined. These are created for only one-time use. In this way when a person/ patient gets discharged these wearable sensors will be disposed. Medical teams can monitor health of the people at while sitting at a distant location keeping a safe distance.

These days we use wireless wearable sensors for monitoring patient health. Wireless Wearable sensors are used to measure physiological conditions of patient. There are some recommended sensors like pulse sensor, respiratory sensor and sensor to measure body temperature which are primarily important to monitor the vital sign of any patient for determining their health conditions are compared in Table 1. The central node see fig. 1 preferably a smart phone which receives data from other sensor nodes will process the all collective information, may apply some decisions and forward to an external location which could be any wide area network base station like LPWAN.

\subsection{Pulse Sensors}

The most fundamental sign in human body is heartbeat. Doctor and medical centres have been using it from centuries. Heartbeat is used to distinguish different diseases such as cardiac arrest, aspiratory embolisms and vasovagal syncope. Human Heartbeat can be checked from different parts of human body such as chest, wrist, ear cartilage, fingertip and many more.

Reading heartbeat from Ear bones and fingertips give most astounding measure of precision. However, it is not easily wearable. Chest worn is wearable yet wrist sensors are considered to be most pleasing for long wearable framework.

It is viable to utilize PPG sensors for beat detecting. these have ceaselessly been turned out to be valuable for estimating more exact heartbeat rate as compare to other sensors like, ultrasonic and Radio Frequencies sensors. PPG sensors are very cost effective also.

LED operated PPG sensors are one of type which transmits light into the Artery and the amount of light which is not absorbed by the blood are receive by the photodiode. The amount of change in frequency of light is recorded and thus pulse rate is measured in recent technologies. In [1], PPG sensors are utilized to count heart beat rate and oxygen in blood. The amount of accuracy in pulse reading is affected by the motion of the person. However, we can use this technology because the people who are quarantined are not subjected to cardiological exercises.

\subsection{Respiratory Rate Sensors}

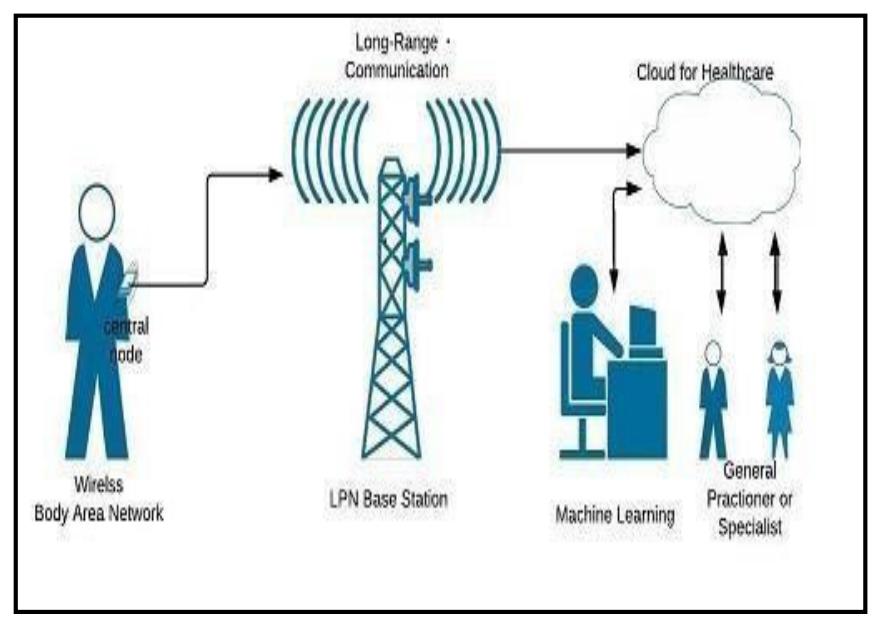

Respiratory monitoring is useful for determining various conditions such as asthma attacks, Apnea episodes, lung cancer hyperventilation due to panic attacks, obstruction of the airways, tuberculosis, etc. The various research works has been done to developed a sensor for measuring respiratory rate due to its importance in various health conditions.

The thermistor based nasal sensor, as used in [5]. AS air exhaled is warmer than the room temperature. This technology effectively senses variations in temperature to measure the respiratory rate. This technology works excellently but accuracy is compromised by the effect of outside temperature fluctuations and it is also not comfortable to wear and roam. ECG derived respiration (EDR) is used in [6] this method works reasonably well to detect respiratory rate but has certain drawbacks like this is not wearable for long time as ECG contacts would likely to cause irritation to the skin and ECG contacts are need to replaced regularly. In [7] uses a Microphone to detect respiratory rate. In this work a wheezing detection a common symptom in asthmatic patient is focused. But microphone is highly susceptible to any external noise and this limits its use in noisy environment and also not wearable for long time. A fiber optic sensor used in one study [8] and was sensitive to measure vibration caused by humans during respiration process. In [9] explains about pressure measuring sensor was developed. Two parallel plate capacitors were used and during inhalation and exhalation, the plated moves apart and then closer allowing the measurement of respiratory rate. This method gives more accurate results as compared to nasal sensor and also comfortable to wear. Here, pressure sensor again highly sensitive to noise. Such as walking into wind but still it will be used in quarantine centers to monitor health. 
Website: ijetms.in Issue:4, Volume No.4, July-2020 DOI: 10.46647/ijetms.2020.v04i04.011

A stretch sensor is commonly used for measuring respiratory rate was done in [10] a stretch sensor made from ferroelectric polymer transducer. When tensile force is applied it generated a charge. Thus, the measurement of changes in this charge enable us to find respiratory rate in [11] change in resistance was calculated to find respiratory rate. Resistance increases on applying the tensile force. The varying resistance causes the change in voltage, this can be further used in calculating breathing rate. This sensor has also been limited by movement as it can cause sensor to sensitize with tensile force because of this, sensor cannot measure accurately when subject is moving or exercising. However, in body area network the main factor to select the sensor type is wear ability. Therefore, stretch sensor is better choice.

These sensors will only be given to those people who have history of respiratory diseases in this way cost can be regulated.

\subsection{Body Temperature Sensor}

Body temperature is the third useful sign to measure the patient condition, it is mostly used to detect fevers, hypothermia, heatstroke and many more.

For measurement of body temperature generally thermistor type sensors are mostly used nowadays. Device is constructed in such a way that it has negative-temperature coefficient (resistance decreases when temperature increases). Positive temperature coefficient is used in [12]. In all recent work thermistor is used to measure a suitable range of temperature of human body, with acceptable amount of error. LED light used temperature measuring guns are very useful in quarantined situations.

\begin{tabular}{|l|l|l|}
\hline Sensor Name & Technique used & Application \\
\hline PPG Sensor & $\begin{array}{l}\text { LED light } \\
\text { intensities }\end{array}$ & $\begin{array}{l}\text { Measure pulse rate } \\
\text { and blood oxygen }\end{array}$ \\
\hline $\begin{array}{l}\text { Nasal Sensor } \\
\text { FDR Sensor }\end{array}$ & $\begin{array}{l}\text { Thermistor } \\
\text { ECG Signal } \\
\text { Pressure-type } \backslash 8 \\
\begin{array}{l}\text { Sensor } \\
\text { Stretch Sensor }\end{array}\end{array}$ & $\begin{array}{l}\text { Elastic Substrate } \\
\text { Parallel plate } \\
\text { capacitor } \\
\text { Ferroelectric } \\
\text { attacks, apnea } \\
\text { episodes, lung } \\
\text { Cancer transducer } \\
\text { hyperventilation, } \\
\text { tuberculosis and } \\
\text { more. }\end{array}$ \\
\hline $\begin{array}{l}\text { Body Temperature } \\
\text { Sensor }\end{array}$ & $\begin{array}{l}\text { Thermistor } \\
\text { Detect } \\
\text { hypothermia, } \\
\text { heatstroke etc. }\end{array}$ \\
\hline
\end{tabular}

Table 1: Comparison of body sensors

\section{Communication Standards}

In healthcare industry, short range communication is used in body area network to communicate sensors with the central node(this will be installed outside of the quarantined centers).
To choose short-range communication standards in health care industry certain requirements are need to fulfil. Firstly, the effect on human health, security and latency. There should not be any adverse effect on human body as it may cause additional concern for patient health. Security of sensitive data of patient is equally important so that it could not be accessed by hacker. We need to build that are systems non pervasive. Finally, in healthcare applications devices low latency is very important. So that system may call an ambulance on emergencies. In such systems, time delay or high latency rate cannot be tolerated as it is a matter of someone's life.

\begin{tabular}{|c|c|c|c|}
\hline $\begin{array}{c}\text { Communication } \\
\text { Standard }\end{array}$ & Range & $\begin{array}{c}\text { Latency } \\
\text { Rate }\end{array}$ & $\begin{array}{c}\text { Power } \\
\text { Consumption }\end{array}$ \\
\hline BLE & High $(150 \mathrm{~m})$ & low & low \\
\hline ZigBee & Short $(30 \mathrm{~m})$ & high & high \\
\hline
\end{tabular}

Table 2: comparison between short-ranging communication standards.

There are many short-ranging communication standards available in this field of IoT, but commonly used technology in the field is Bluetooth enabled with low energy i.e. BLE and ZigBee standard. Differences between them are listed in table 2. According to table 2, we can say that BLE is suitable for healthcare and medical applications. It is secure, Non pervasive, not very expensive, covers very high range, low maintenance cost, low-latency(Time-delay) rate and that best thing with this technology is that it has low power consumption. BLE was developed by special interest group aiming to enable seamless Bluetooth service for IoT and the group focused on low cost and high quality delivering standard. BLE uses star topology which we have discussed earlier that it is perfect for healthcare application. In body area network a central node act as a centre of the star topology with all sensors linked with it. BLE

communicate up to $150 \mathrm{~m}$ in an open area, sufficient for healthcare system WBAN as discussed in [13]. It also has a low latency rate of $3 \mathrm{~ms}$ and high data rate of $1 \mathrm{Mbps}$ as discussed in [15]. For security features BLE uses 128-AES encryption to protect sensitive data from hackers.

LPN (Low-power Network ) or also commonly known as low-power wide-area network is a long-range communication standard suitable for IoT application. The range of LPN is longer than the traditionally used IoT communication methods. LPN is created to make use short bust of data which has an advantage over $3 \mathrm{G}$ cellular network. As named low power network allows design for low power devices, which ensures that installed healthcare devices will operate for a longer time. This reduces the threat of patient being offline for longer period of time. The features of these two are shown in table 3. Therefore, LPN is highly suitable for numerous healthcare and medical areas such as monitoring of 
Website: ijetms.in Issue:4, Volume No.4, July-2020 DOI: 10.46647/ijetms.2020.v04i04.011

critical health of patient, receiving and attending emergency calls and receiving real time updates without any disruption. Based on mentioned advantages LPN is best for transmitting data to the cloud for storage and processing.

Table 3. Communication standards

\begin{tabular}{|l|l|l|l|}
\hline $\begin{array}{l}\text { Communication } \\
\text { Standard (Long } \\
\text { Range) }\end{array}$ & $\begin{array}{l}\text { Operation } \\
\text { Band }\end{array}$ & Range & Data Rate \\
\hline LoRaWAN & GSM & $7.2 \mathrm{Km}$ & $\begin{array}{l}0.25- \\
5.5 \mathrm{Kbps}\end{array}$ \\
\hline NB-IoT & LTE & $15 \mathrm{Km}$ & $250 \mathrm{Kbps}$ \\
\hline
\end{tabular}

The most useful standard for LPNs are Sigfox, LoRaWAN and NBIoT. The new emerging standard Narrowband IoT influencing more to HealthIIoT over other two standards of LPN.NB-IoT can operate in LTE bands [15]. Due to high receiver sensitivity it covers the area up to $15 \mathrm{~km}$ with high speed [16]. 3GPP(S3) protection scheme is used by NBIoT including other security features like authentication, securing user and device identity and data integrity [17]. Therefore, NBIoT is appropriate for healthcare purpose.

\section{Cloud for Healthcare}

Cloud technologies can be used to data storage and processing. Also, family members of quarantined people can be given access to the data of their loved ones. These data can be used by various government authorities for decision making. India has many available cloud storages and these can be used to better decision making in these unpredictable scenarios.

\section{HealthIIoT System Design}

Communication System available till now, we have studies most of the IoT technologies used in HealthIIoT. The designs and frameworks for designing a seamless communication channel for HealthIIoT network are:-

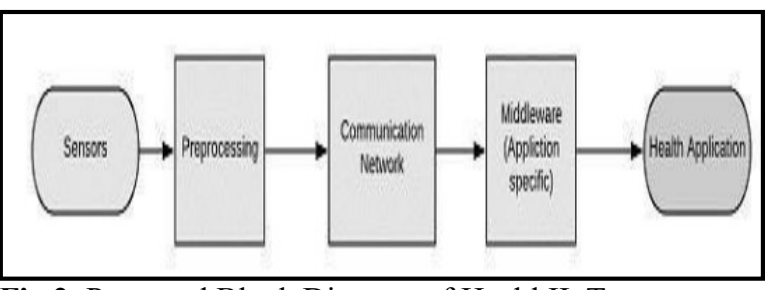

Fig 2: Proposed Block Diagram of HealthIIoT

The first considerations the design of sensors for BSN, which helps in interacting with physical environment, and they must have capability of storing and processing health monitoring data of patient intelligently at the edge of the network itself, called a pre-processing refer. Fig.2. The choice of PPG sensor is best for pulse sensing, stretch sensor for measuring respiratory rate and thermistor-based sensor for measuring body temperature for healthcare industry.

The next important design issue is choosing a right communication network in mainly, terms of Power requirement by IoT nodes. It has concluded that use of LPN is suitable choice for designing HealthIIoT communication network.it is therefore BLE for short range communication and NB-IOT is suitable to use for healthcare application Let us than come to cross layer communication on heterogeneous devices requires middleware platform

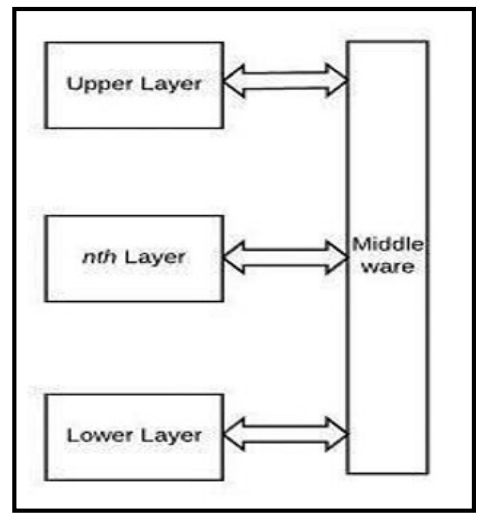

Fig 3: Conceptual illustration of Centralized Middleware

Service oriented IoT middleware used for HealthIIoT are based on Service Oriented Architecture (SOA) which provides specific services like providing patient health data to the doctors for reference and recommendations. HYDRA is a one type of service oriented middleware.it consists of many software components for application of healthcare it is very useful to handle many task solutions see fig 3 . Ubiquitous computing is the necessary objective to connect healthcare system with Internet of things. Interoperability of such devices needs standardization of heterogeneous system. Middleware act as sandwich software between devices and applications to provide specific service to developers so that they focus more on quality of service.

Hence, Middleware provides an effective opportunity for building Application Programming Interface (API).

HealthIIoT middleware faces many challenges like Interoperability, scalability, big data analytics, context detection, security and privacy. In [12] Hydra middleware provides interoperable access to data information and knowledge across heterogeneous supported both new and existing wired or wireless network which operated with limited resources like computing power, energy and memory usage which would be beneficial for real time usage in HealthIIoT. It is very effective for health application by providing secure, trust worthy and fault tolerance which all are 
Website: ijetms.in Issue:4, Volume No.4, July-2020 DOI: 10.46647/ijetms.2020.v04i04.011

the very key component of effective health monitoring and providing appropriate solution.

Finally, comes a health application layer, this includes creating an EHR(electronic health record) and transmit warning in case any abnormal ties are found. machine learning algorithms are may useful to fix minor problems and suggest a prescription to the patient.

\section{Conclusion and Future work}

The IoT based seamless communication channel for healthcare system is designed in order to help doctors to monitor patient critical health conditions. We than present a comparative study relating to each block of the proposed model. various body sensors are studied mainly those who focuses on monitoring vital signs. Communication standards are then compared for both short- and long-range communication based on the suitability for healthcare Industry. Cloud technologies is used for data storage in healthcare and then Proposed a model for seamless communication of various technologies used in health IoT.

Based on our study over various technologies in the area of IoT such as wearable Sensors, various communication standards and cloud computing, and machine learning. We explored different areas for future research. The security and using machine learning in health IoT provide opportunity for researchers in the area Industrial health IoT.

\section{Reference}

[1] N.Zhu et al.," bridging e-health and the internet of things: the SPHERE project," IEEE Intell. Syst.,vol.30, 4, pp.39-46, Jul./Aug.2015

[2] P.Gope and T.Hwang, "BSN-care : A secure IoTbased modern healthcare system using body sensor network," IEEE Sensors J., vol. 16, no.5, pp. 1368-1376, Mar. 2016

[3] J.enko, M. Kos, and I. Kramberger, "Pulse rate variability and blood oxidation content identification using miniature wearable wrist device, 'in Proc. Int. Conf. Syst., Signals Image Process. (IWSSIP), May 2016, pp. $1-4$

[4] H. Lee, H. Ko, C. Jeong, and J. Lee, "Wearable photoplethysmographic sensor based on different LED light intensities," IEEE Sensors J., vol. 17, no. 3, pp. 587_588, Feb. 2017.

[5] S. Milici, J. Lorenzo, A. Lázaro, R. Villarino, and D. Girbau, 'Wireless breathing sensor based on wearable modulated frequency selective surface,"IEEE Sensors J., vol. 17, no. 5, pp. 1285_1292, Mar. 2017.
[6] C. Varon, A. Caicedo, D. Testelmans, B. Buyse, and S. van Huffel, "A novel algorithm for the automatic detection of sleep apnea from singlelead ECG," IEEE Trans. Biomed. Eng., vol. 62, no. 9, pp. 2269_2278, Sep. 2015.

[7] Mahesh, Bhasutkar, Maninti Venkateswarlu, and M. Raghavendra. "End-to-end congestion control techniques for Router." 2011 International Conference on Communication Systems and Network Technologies. IEEE, 2011.

[8] Mahesh, B., and K. Shyam Sunder Reddy. "Router Aided Congestion Control Techniques." Second International Conference on Information Systems and Technology.

[9] Mahesh, B. "Dynamic Update and Public Auditing with Dispute Arbitration for Cloud Data." Journal of Advanced Database Management \& Systems 4.3 (2017): 14-19.

[10] Mahesh, B., et al. "A Review on Data Deduplication Techniques in Cloud." Embedded Systems and Artificial Intelligence. Springer, Singapore, 2020. 825-833.O. Atalay,W. R. Kennon, and E. Demirok, "Weft-knitted strain sensor for monitoring respiratory rate and its electromechanical modeling," IEEE Sensors J., vol. 15, no. 1, pp.110_122, Jan. 2015.

[11] M. Eisenhauer, P. Rosengren, and P. Antolin, "A development platform for integrating wireless devices and sensors into ambient intelligence systems," in Proceedings of the 6th IEEE Annual Communications Society Conference on Sensor, Mesh and Adhoc Communications and Networks Workshops (SECON Workshops'09), pp. 1-3, IEEE, Rome, Italy. June 2009.

[12] K.-H. Chang, "Bluetooth: A viable solution for IoT? [Industry Perspectives],"IEEE Wireless Commun., vol. 21, no. 6, pp. 6 7, Dec. 2014.

[13] Bluetooth. (2009). SIG Introduces Bluetooth Low Energy Wireless Technology, the Next Generation Bluetooth Wireless Technology [Online]

[14] R. Ratasuk, B. Vejlgaard, N. Mangalvedhe, and A. Ghosh, "NB-IoT system for M2M communication," in Proc. IEEE Wireless Communication. Netw. Conf., Apr. 2016, pp. 1_5.

[15] S. Landstrm, J. Bergstrm, E. Westerberg, D. Hammarwall, "NB-IoT: A sustainable technology for connecting billions of devices", in Ericsson Technology Review, vol. 93, no. 3, pp. 2-11, April 2016

[16] Wu, Longfei, et al. "Access Control Schemes for Implantable Medical Devices: A Survey." IEEE Internet of Things Journal(2017)

[17] F. Corno, L. De Russis, and A. M. Roffarello, "A healthcare support system for assisted living facilities: An iot solution," in Computer Software and Applications Conference (COMPSAC), 2016 IEEE 40th Annual, vol. 1. IEEE, 2016, pp. 344-352

[18] Baker, S., Xiang, W. and Atkinson, I. (2017). Internet of Things for Smart Healthcare: Technologies, Challenges, and Opportunities. IEEE Access, 5, pp.26521-26544.

[19] Zhang, W., Kumar, M., Yu, J. and Yang, J. (2018). Medical longdistance monitoring system based on internet of things. EURASIP Journal on Wireless Communications and Networking, 2018(1).

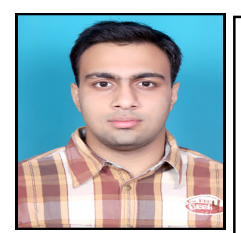

Mr. Ishan Bhatt is an MBA in sales and marketing with minor in finance. He has completed his Bachelor in technology degree in Computer science and Engineering. His area of interest is in Marketing, IoT, Networking, Machine learning and data analytics.

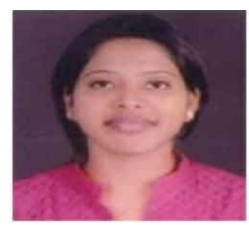

Ms. Shweta Yadav is Master in technology in electronics and communication. Her area of interest is in communication, networking and antenna 